\title{
Symmetric Masking Function of Segments
}

\author{
Péter Lukács
}

(Communicated by Kazım İlarslan)

\begin{abstract}
We show that the masking function of two segments on a surrounding circle $\mathcal{C}$ is symmetric to a straight line $\sigma$ passing through the centre of $\mathcal{C}$ if and only if the set of the segments is also symmetric to $\sigma$.
\end{abstract}

Keywords: Visual angle; masking number; masking function; geometric tomography.

AMS Subject Classification (2020): 44A12; 52A10.

\section{Introduction}

When a light beam passes through thin light-transmitting membranes, for example through bubbles, the amount of the absorbed light depends on the permeability and the number of membranes. If there is only one membrane, the incoming light intensity at an external point $P$ depends only on the degree of the visual angle the membranes subtends. If there are multiple membranes, the intensity depends on the sum of these visual angles. This sum is the masking number [5] of the given set of membranes at point $P$. The function that assignes the masking numbers to points is called masking function.

The general question in this article is that if the masking function of some segments in the plane is known on a circle $\mathcal{C}$ surrounding the segments, then how to determine the segments. Specificaly we consider the problem for two segments and we show that the masking function of two segments restricted to a surrounding circle $\mathcal{C}$ is symmetric to a straight line $\sigma$ passing through the centre of the circle if and only if the set of the segments is also symmetric to $\sigma$.

\section{Notations and preliminaries}

Let $\boldsymbol{g}:[0,1] \rightarrow \mathbb{R}$ be a differentiable curve parameterized by arc length. Let $\boldsymbol{t}(s), \boldsymbol{n}(s)$ and $\kappa(s)$ be the unit tangent vector, the normal vector and the curvature of $\boldsymbol{g}$ at $\boldsymbol{g}(s)$, respectively. Let $L(s)$ and $R(s)$, respectively, be the point of tangency of the left and right side tangent lines of the strictly convex domain $\mathcal{K}$, passing through the point $\boldsymbol{g}(s)$. Let $\alpha(s)$ and $\beta(s)$ be the angle of vectors $\overrightarrow{\boldsymbol{g}(s) L(s)}$ and $\overrightarrow{\boldsymbol{g}(s) R(s)}$ made with vector $\boldsymbol{t}(s)$ respectively, and finally let $l(s)$ and $r(s)$ be the length of the mentioned vectors, respectively.

Lemma 2.1. ([4, Lemma 1]) Angle $\alpha$ is differentiable, and $\dot{\alpha}(s)=\frac{\sin \alpha(s)}{l(s)}-\kappa(s)$.

As a consequence, the right derivative of $\nu_{\mathcal{K}}(s)=\alpha(s)-\beta(s)$ is

$$
\dot{\nu}(s+)=\frac{\sin \alpha(s)}{l(s)}-\frac{\sin \beta(s)}{r(s)} .
$$

Now let $\mathcal{K}$ be a segment $\mathcal{S}=\overline{A B}$ surrounded by the circle $\mathcal{C}$ on the chord $\mathcal{H}=\overline{\boldsymbol{g}\left(s_{1}\right) \boldsymbol{g}\left(s_{2}\right)}$, where $\boldsymbol{g}$ is a parametrization of $\mathcal{C}$ by are length.

Lemma 2.2. ([6, Theorem 3.4]) Values $\dot{\nu}_{\mathcal{S}}\left(s_{1} \pm\right)$ and $\dot{\nu}_{\mathcal{S}}\left(s_{2} \mp\right)$ determine $\mathcal{S}$. 
Proof. Without loss of generality, we can assume the radius of the circle $\mathcal{C}$ is such that the length of the chord $\mathcal{H}$ is 2 .

Let the signed distance of points $A$ and $B$ from the midpoint $H$ of $\mathcal{H}$ respectively be $a$ and $b$, such that $-1<b<a<1$. Let $\xi$ be the angle made by $\boldsymbol{t}\left(s_{1}\right)$ and $\mathcal{H}$.

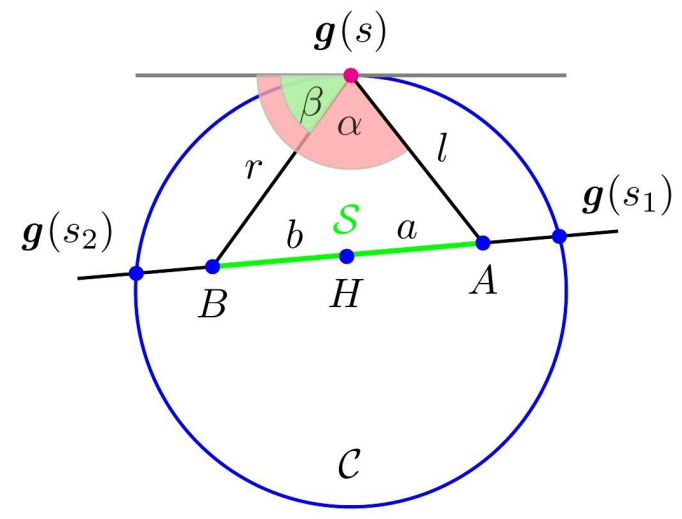

According to formula (2.1) we have

$$
\left|\dot{\nu}\left(s_{1}+\right)\right|=\left|\frac{\sin \xi}{1-a}-\frac{\sin \xi}{1-b}\right| \text { and }\left|\dot{\nu}\left(s_{2}+\right)\right|=\left|\frac{\sin (\pi-\xi)}{1+a}-\frac{\sin (\pi-\xi)}{1+b}\right| .
$$

Let

$$
x:=\frac{\left|\dot{\nu}\left(s_{1}+\right)\right|}{\sin \xi} \text { and } y:=\frac{\left|\dot{\nu}\left(s_{2}+\right)\right|}{\sin (\pi-\xi)} .
$$

Then

$$
x=\frac{1}{1-a}-\frac{1}{1-b}=\frac{a-b}{(1-a)(1-b)} \quad \text { and } \quad y=\frac{1}{1+b}-\frac{1}{1+a}=\frac{a-b}{(1+a)(1+b)}
$$

are positive, and $x \neq \frac{1}{1-a}$ because otherwise $\frac{1}{1-b}=0$ follows.

According to the first equation in (2.2) we have $b=\frac{a-x(1-a)}{1-x(1-a)}$. From the second equation of (2.2), after substitution of $b$, we get

$$
a^{2}(x-2 x y-y)+a(-2 x-2 y)+(x+2 x y-y)=0,
$$

the roots of which are

$$
a_{1}=\frac{x+y-2 \sqrt{x y+x^{2} y^{2}}}{x-y-2 x y} \text { and } a_{2}=\frac{x+y+2 \sqrt{x y+x^{2} y^{2}}}{x-y-2 x y} .
$$

Due to the triangular inequality

$$
\left|x+y+2 \sqrt{x y+x^{2} y^{2}}\right|=|x|+|y|+\left|2 \sqrt{x y+x^{2} y^{2}}\right|>|x|+|y|+|2 x y|>|x-y-2 x y|,
$$

$\left|a_{2}\right|>1$ follows, so $a_{1}$ is the only solution, hence

$$
a=a_{1} \quad \text { and } \quad b=\frac{x+y-2 \sqrt{x y+x^{2} y^{2}}}{x-y+2 x y},
$$

that proves the lemma.

Notice that, by the last formula, $\mathcal{S}$ is symmetric to the midpoint of $H$, if $\dot{\nu}_{\mathcal{S}}\left(s_{1} \pm\right)=\dot{\nu}_{\mathcal{S}}\left(s_{2} \mp\right)$.

Lemma 2.3. There is at most one segment $\hat{\mathcal{S}}$ different from segment $\mathcal{S}$, lying on the same chord $\mathcal{H}$ as $\mathcal{S}$, that satisfies

(1) $\dot{\nu}_{\mathcal{S}}\left(s_{1}+\right)=\dot{\nu}_{\hat{\mathcal{S}}}\left(s_{1}+\right)$ and $\nu_{\mathcal{S}}\left(s_{3}\right)=\nu_{\hat{\mathcal{S}}}\left(s_{3}\right)$, or

(2) $\nu_{\mathcal{S}}\left(s_{4}\right)=\nu_{\hat{\mathcal{S}}}\left(s_{4}\right)$ and $\nu_{\mathcal{S}}\left(s_{3}\right)=\nu_{\hat{\mathcal{S}}}\left(s_{3}\right)$,

where $\boldsymbol{g}\left(s_{3}\right)$ and $\boldsymbol{g}\left(s_{4}\right)$ is not on $\mathcal{H}$. 
Proof. Let $T$ be the perpendicular projection of the point $\boldsymbol{g}\left(s_{3}\right)$ onto $\mathcal{H}$. Let $l$ and $k$ be, respectively, the distances of $\boldsymbol{g}\left(s_{3}\right)$ and $\boldsymbol{g}\left(s_{4}\right)$ from $T$ respectively. Let $a$ and $b$ be the signed distances of points $A$ and $B$ from $T$, respectively.

We clearly have $\nu_{\mathcal{S}}\left(s_{3}\right)=\arctan \frac{b}{l}-\arctan \frac{a}{l}$, and $\nu_{\mathcal{S}}\left(s_{4}\right)=\arctan \frac{b}{k}-\arctan \frac{a}{k}$, hence

$$
x:=\tan \nu_{\mathcal{S}}\left(s_{3}\right)=\tan \left(\arctan \frac{b}{l}-\arctan \frac{a}{l}\right)=\frac{(b-a) l}{l^{2}+a b}>0
$$

and

$$
y:=\tan \nu_{\mathcal{S}}\left(s_{4}\right)=\frac{(b-a) k}{k^{2}+a b}>0
$$

From formula (2.1) we obtain $z:=\frac{\left|\dot{\nu}\left(s_{1}+\right)\right|}{\sin \xi}=\frac{1}{m-b}-\frac{1}{m-a}$, where $m$ is the distance of $T$ from $g\left(s_{1}\right)$, that gives

$$
m^{2} z-b m z-a m z+a b z=b-a .
$$

If (1) is valid, then (2.3) and (2.5), if (2) is valid, then (2.3)) and (2.4) determine at most two pairs $(a, b)$ as solutions.

In the plane, the closed set of points where two convex domains subtend equal angles is called equioptic, and the closed set of points where they subtend supplementary angles are called compoptic.

Theorem 2.1. ([3, Lemma 2.1]) The union of the equioptic and compoptic sets of two segments is two algebraic curves of order three.

These two curves are called the Apollonios curves. Unfortunately, the equation for these curves does not show which parts belong to the equioptic or compoptic, but for collinear segments we have a description.

Lemma 2.4. ([6, Lemma 2.3]) Let $\mathcal{S}_{1}$ and $\mathcal{S}_{2}$ be segments on a straight line $f$.

(i) If $\mathcal{S}_{1}$ and $\mathcal{S}_{2}$ have different lengths, then their Apollonian curves are the union of straight line $f$ and a (possibly degenerate) circle $\mathcal{C}$, one of which is part of the equioptic and the other one is part of the compoptic.

(ii) If $\mathcal{S}_{1}$ and $\mathcal{S}_{2}$ are of the same length, then their Apollonian curves are the union of $f$ and a straight line g, or a possibly degenerate circle $\mathcal{C}$, both symmetric to $f$, where $g$ is part of the equioptic, and $\mathcal{C}$ is part of the compoptic.

Corollary 2.1. ([6, Corollary 2.4])

(1) If the lengths of the collinear segments are different, then

(1.a) if one of the segments contains the other, then there is no equioptic circle,

(1.b) if they do not contain each other and have a common point, then their equioptic circle passes through a given common point of the segments,

(1.c) if they are disjoint, then their equioptic circle passes through $f$ between the nearest endpoints of the segments.

(2) If the lengths of the collinear segments are equal, then their equioptic straight line is the symmetry axis of the two segments.

Lemma 2.5. If two segments are on the same chord of a circle $\mathcal{C}$, and there are three different points on the cirlce $\mathcal{C}$, where they subtend equal nonzero angles, then the segments coincide.

Proof. Suppose that $\mathcal{S}_{1} \not \equiv \mathcal{S}_{2}$. Then $\mathcal{C}$ intersects in three points equioptic of the two segments. However, according to Lemma 2.4 the equioptic is a circle or a straight line, and a straight line can only intersect $\mathcal{C}$ in two points, hence the equioptic contains $\mathcal{C}$. Then, with elementary tools it is easy to verify that the two segments coincide [1, Lemma 2.1].

\section{Masking function of two segments on a circle}

Let $\mathcal{S}_{1}=\overline{A B}$ and $\mathcal{S}_{2}=\overline{C D}$ be segments inside the circle $\mathcal{C}$ and let $\tau_{\mathcal{S}_{1}, \mathcal{S}_{2}}=\nu_{\mathcal{S}_{1}}+\nu_{\mathcal{S}_{2}}$ be their masking function, restricted to the circle $\mathcal{C}$.

Theorem 3.1. Fuction $\tau_{\mathcal{S}_{1}, \mathcal{S}_{2}}$ is symmetric on circle $\mathcal{C}$ to a straight line $\sigma$ passing through the center of circle $\mathcal{C}$ if and only if the segments are both symmetrical to $\sigma$, or they are reflections of each other to $\sigma$. 
Proof. It is evident that the symmetry of $\tau_{\mathcal{S}_{1}, \mathcal{S}_{2}}$ follows from both conditions. Now suppose that $\tau_{\mathcal{S}_{1}, \mathcal{S}_{2}}$ is symmetric. The proof goes step-by-step according to the number of the roots of $\tau_{\mathcal{S}_{1}, \mathcal{S}_{2}}$.

The masking function has no roots.

Let $\boldsymbol{g}\left(s_{i}\right)(i=1,2,3,4)$ be the points on the circle, where $\tau_{\mathcal{S}_{1}, \mathcal{S}_{2}}$ is not differentiable. Consider the left and right derivatives of $\tau_{\mathcal{S}_{1}, \mathcal{S}_{2}}$ at these points, and observe that $\nu_{\mathcal{S}_{1}}$ and $\nu_{\mathcal{S}_{2}}$ are differentiable outside the points $\boldsymbol{g}\left(s_{i}\right)$ $(i=1,2,3,4)$, but at any $s_{i}$ exactly one of $\nu_{\mathcal{S}_{j}}(j=1,2)$ is not differentiable and the left and right derivative of that $\nu_{\mathcal{S}_{j}}$ are counterparts of each other, so $\dot{\tau}_{\mathcal{S}_{1}, \mathcal{S}_{2}}\left(s_{i}+\right)+\dot{\tau}_{\mathcal{S}_{1}, \mathcal{S}_{2}}\left(s_{i}-\right)$ is twice the respective derivative $\dot{\mathcal{S}}_{\mathcal{S}_{j}}\left(s_{i} \pm\right)$. These derivatives $\dot{\nu}_{\mathcal{S}_{j}}\left(s_{i} \pm\right)$ and the chords of the segments clearly determines the segments by Lemma 2.2.

(a) No $\boldsymbol{g}\left(s_{i}\right)(i=1,2,3,4)$ is on the $\sigma$ axis.

We can assume that $\boldsymbol{g}\left(s_{1}\right)$ and $\boldsymbol{g}\left(s_{4}\right)$ are on the right side of the $\sigma$, and $\boldsymbol{g}\left(s_{2}\right)$ and $\boldsymbol{g}\left(s_{3}\right)$ are the reflections of them, respectively. Then the segments can only be in three different positions (see Figure 1).

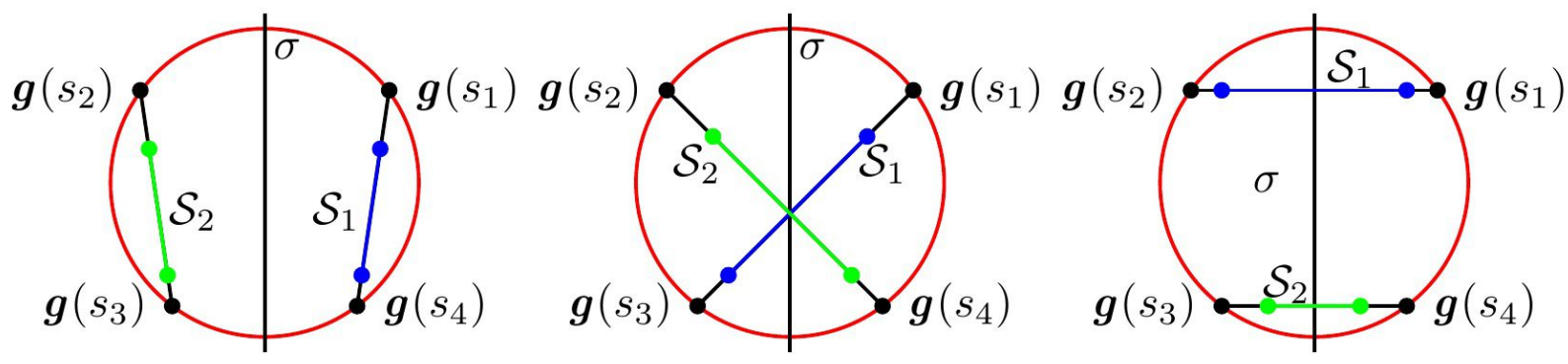

Figure 1. Possible positions of the segments.

Due to the symmetry of $\tau_{\mathcal{S}_{1}, \mathcal{S}_{2}}$, the respective derivatives $\dot{\nu}_{\mathcal{S}_{j}}\left(s_{i} \pm\right)$ are equal at the reflected points, therefore the segments are either the reflections of each other, or, in the last case, they are symmetrical with respect to $\sigma$.

(b) Two points, say $\boldsymbol{g}\left(s_{1}\right)$ and $\boldsymbol{g}\left(s_{3}\right)$ are on $\sigma$.

Then $\boldsymbol{g}\left(s_{2}\right)$ is the reflection of $\boldsymbol{g}\left(s_{4}\right)$ with respect to $\sigma$, and the segments can only be in two different positions (see Figure 2).
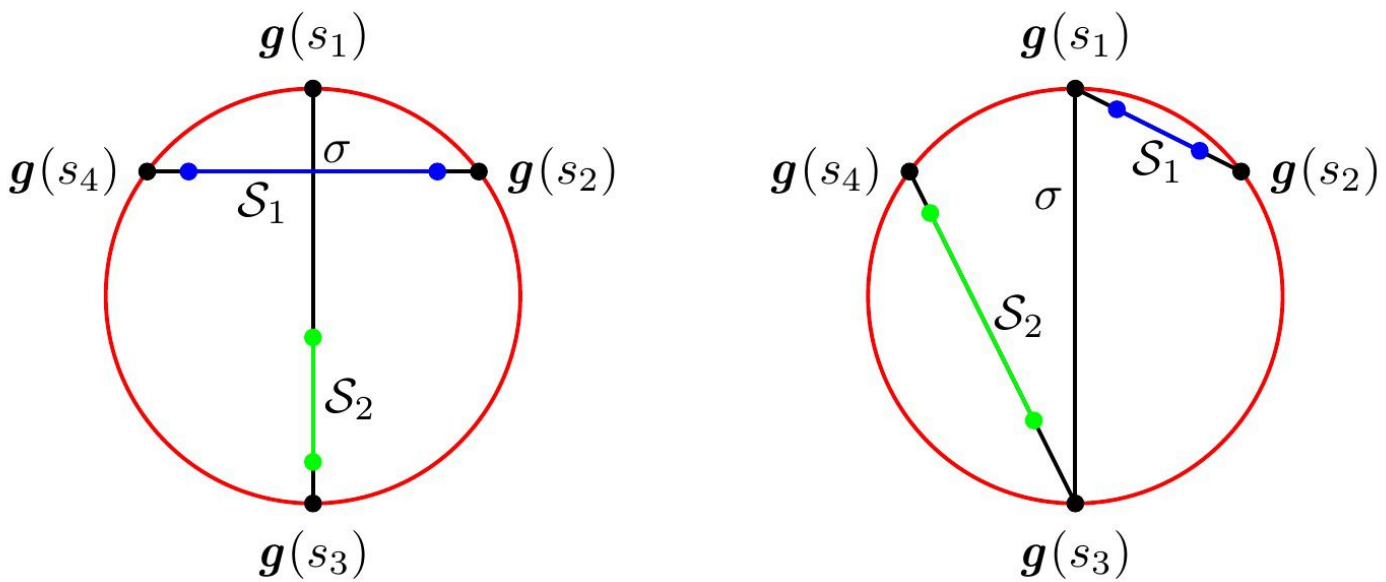

Figure 2. Possible positions of the segments in case (b).

In the first case the symmetry is easily comes from Lemma 2.2 .

The second case, where neither of the segments lies on $\sigma$, is impossible by the following reason: By the symmetry, $\tau_{\mathcal{S}_{1}, \mathcal{S}_{2}}$ has local extremums at $\boldsymbol{g}\left(s_{1}\right)$ and $\boldsymbol{g}\left(s_{3}\right)$. As $\nu_{\mathcal{S}_{1}}$ is minimal at $\boldsymbol{g}\left(s_{3}\right)$ and $\nu_{\mathcal{S}_{2}}$ is minimal at $\boldsymbol{g}\left(s_{1}\right)$, we deduce that $\nu_{\mathcal{S}_{1}}$ is maximal at $\boldsymbol{g}\left(s_{3}\right)$ and $\nu_{\mathcal{S}_{2}}$ is maximal at $\boldsymbol{g}\left(s_{1}\right)$. Now, let us examine the right and left derivatives of $\tau_{\mathcal{S}_{1}, \mathcal{S}_{2}}$ in $s_{2}$ and $s_{4}$. In $s_{2}$ the value of the derivative towards $s_{1}$ is greater than towards $s_{3}$, as 
the value of $\nu_{\mathcal{S}_{2}}$ increases towards local maximum at $s_{1}$ and decreases towards $s_{3}$. In point $s_{4}$, with a similar way of thinking, we can deduce the reverse, so here the derivative is higher towards $s_{3}$ than towards $s_{1}$, which contradicts the symmetry.

The masking function has one root.

Let $\boldsymbol{g}\left(s_{3}\right)$ be the only root of $\tau_{\mathcal{S}_{1}, \mathcal{S}_{2}}$ that is therefore on $\sigma$. Let $\boldsymbol{g}\left(s_{1}\right)$ and $\boldsymbol{g}\left(s_{2}\right)$ be points of the cirlce outside $\sigma$, where $\tau_{\mathcal{S}_{1}, \mathcal{S}_{2}}$ is not differentiable. Then $\boldsymbol{g}\left(s_{1}\right)$ is the reflection of $\boldsymbol{g}\left(s_{2}\right)$ in $\sigma$, so $\dot{\nu}_{\mathcal{S}_{1}}\left(s_{1} \pm\right)=\dot{\nu}_{\mathcal{S}_{2}}\left(s_{2} \mp\right)=$ $\left(\dot{\tau}_{\mathcal{S}_{1}, \mathcal{S}_{2}}\left(s_{1}+\right)-\dot{\tau}_{\mathcal{S}_{1}, \mathcal{S}_{2}}\left(s_{1}-\right)\right) / 2$ and by Lemma 2.3 , these derivatives with $\nu_{\mathcal{S}_{2}}\left(s_{1}\right)=\nu_{\mathcal{S}_{1}}\left(s_{2}\right)=\tau_{\mathcal{S}_{1}, \mathcal{S}_{2}}\left(s_{1}\right)$ allow only two possible segments on each straight line $f_{1}:=A B$ and $f_{2}:=C D$. Let these segments $\mathcal{S}_{1}, \tilde{\mathcal{S}}_{1}$ on $f_{1}$, and $\mathcal{S}_{2}, \tilde{\mathcal{S}}_{2}$ on $f_{2}$ respectively, where $\mathcal{S}_{1}$ and $\mathcal{S}_{2}$, plus $\tilde{\mathcal{S}}_{1}$ and $\tilde{\mathcal{S}}_{2}$ are reflections of each other with respect $\sigma$ (see Figure 3).

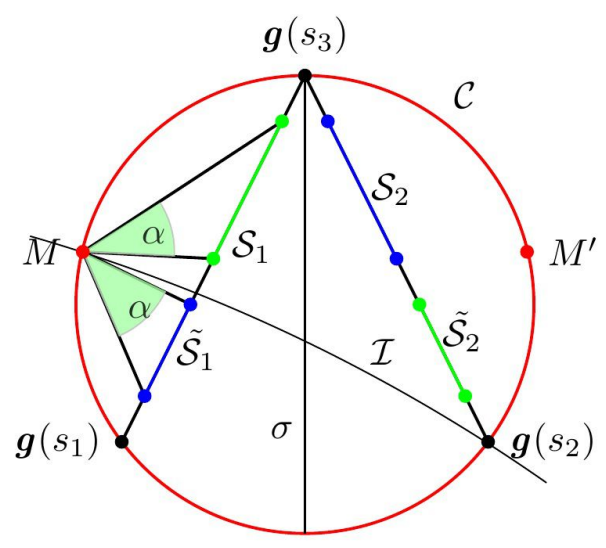

Figure 3. Possible positions of the segments if three roots exist.

If $\tau_{\mathcal{S}_{1}, \tilde{\mathcal{S}}_{2}}$ is symmetric to $\sigma$, then by the symmetry of $\tau_{\mathcal{S}_{1}, \mathcal{S}_{2}}$ also $\tau_{\tilde{\mathcal{S}}_{1}, \mathcal{S}_{2}}$ is symmetrical to $\sigma$, so $\mathcal{S}_{1}$ and $\tilde{\mathcal{S}}_{1}$ subtend the same angle at $\boldsymbol{g}\left(s_{2}\right)$. Thus, $\boldsymbol{g}\left(s_{2}\right)$ is on the equioptics of $\mathcal{S}_{1}$ and $\tilde{\mathcal{S}}_{1}$. This equioptic $\mathcal{I}$ passes through $\boldsymbol{g}\left(s_{2}\right)$ and, by Corollary 2.1, the chord $\overline{\boldsymbol{g}\left(s_{1}\right) \boldsymbol{g}\left(s_{3}\right)}$. As $\tau_{\mathcal{S}_{1}, \mathcal{S}_{2}}$ is symmetric to $f_{1}$, equioptic $\mathcal{I}$ also intersects the circular of $\mathcal{C}$ in the other side of $f_{1}$ than $\boldsymbol{g}\left(s_{2}\right)$. Let this intersection point be $M$.

As $\tilde{\mathcal{S}}_{1}$ and $\mathcal{S}_{1}$ subtend equal visual angles at $M$, the two pairs of the segments also have equal masking numbers in $M$, hence, $\mathcal{S}_{2}$ and $\tilde{\mathcal{S}}_{2}$ subtend also equal angles here.

By the symmetry of $\tau_{\mathcal{S}_{1}, \mathcal{S}_{2}}, \mathcal{S}_{1}$ and $\tilde{\mathcal{S}}_{1}$ subtend also equal angles at $M^{\prime}$, which is the reflection of $M$ to $\sigma$. Therefore, $\mathcal{S}_{1}$ and $\tilde{\mathcal{S}}_{1}$ subtend equal angles at points $\boldsymbol{g}\left(s_{2}\right), M$, and $M^{\prime}$ outside $f_{1}$. By Lemma 2.5, these three values determine the segments, so $\mathcal{S}_{1} \equiv \tilde{\mathcal{S}}_{1}$. Similarly, $\mathcal{S}_{2} \equiv \tilde{\mathcal{S}}_{2}$ follows, that proves the symmetry of the segments.

The masking function has two roots.

Let $\boldsymbol{g}\left(s_{1}\right)$ and $\boldsymbol{g}\left(s_{2}\right)$ be the roots of $\tau_{\mathcal{S}_{1}, \mathcal{S}_{2}}$. Let $a<b$ and $c<d$ be the signed distance of $A, B$ and $C, D$, respectively from the midpoint $H$ of the common chord $\mathcal{H}$ of $\mathcal{S}_{1}$ and $\mathcal{S}_{2}$. The bisector of $\mathcal{H}$ is clearly $\sigma$ and let its intersections with circle $\mathcal{C}$ be $\boldsymbol{g}\left(s_{3}\right)$ and $\boldsymbol{g}\left(s_{4}\right)$. (See Figure 4.)

Let $\xi$ be the angle made by $\boldsymbol{t}\left(s_{1}\right)$ and $\mathcal{H}$, and let $l$ be the distance of $\mathcal{H}$ from $\boldsymbol{g}\left(s_{3}\right)$. Choose the radius of the circle so that the length of chord $\mathcal{H}$ is 2 , so we have $-1<a<b<1$ and $-1<c<d<1$.

Due to formula (2.1) we have

and

$$
\left|\dot{\mathcal{S}}_{\mathcal{S}_{1}, \mathcal{S}_{2}}\left(s_{1}+\right)\right|=\left|\frac{\sin \xi}{h-a}-\frac{\sin \xi}{h-b}+\frac{\sin \xi}{h-c}-\frac{\sin \xi}{h-d}\right|,
$$

$$
\left|\dot{\tau}_{\mathcal{S}_{1}, \mathcal{S}_{2}}\left(s_{2}-\right)\right|=\left|\frac{\sin \xi}{h+a}-\frac{\sin \xi}{h+b}+\frac{\sin \xi}{h+c}-\frac{\sin \xi}{h+d}\right| .
$$

Again from (2.1) we get

$$
\left|\dot{\mathcal{S}}_{\mathcal{S}_{1}, \mathcal{S}_{2}}\left(s_{3}+\right)\right|=\left|\frac{l}{l^{2}+a^{2}}-\frac{l}{l^{2}+b^{2}}+\frac{l}{l^{2}+c^{2}}-\frac{l}{l^{2}+d^{2}}\right|,
$$

because $\left(\sin A g\left(s_{3}\right) H \angle\right)=\frac{l}{\sqrt{a^{2}+l^{2}}},\left(\sin B g\left(s_{3}\right) H \angle\right)=\frac{l}{\sqrt{b^{2}+l^{2}}}$ and so on. 


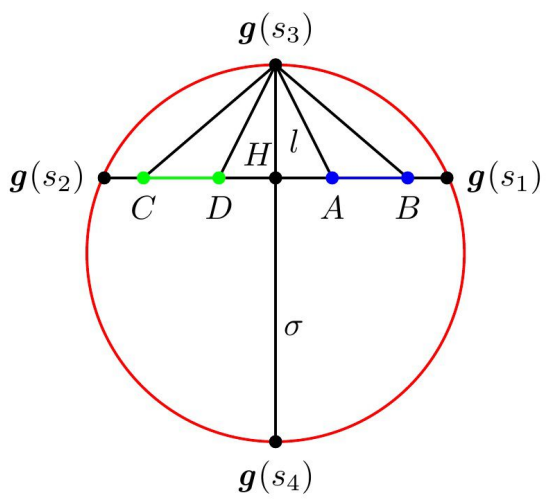

Figure 4. Possible positions of the segments if two roots exist.

Due to the symmetry of $\tau_{\mathcal{S}_{1}, \mathcal{S}_{2}}$ we have $\left|\dot{\tau}_{\mathcal{S}_{1}, \mathcal{S}_{2}}\left(s_{1}+\right)\right|=\left|\dot{\tau}_{\mathcal{S}_{1}, \mathcal{S}_{2}}\left(s_{2}-\right)\right|$, and $\dot{\tau}_{\mathcal{S}_{1}, \mathcal{S}_{2}}\left(s_{3}+\right)=0$, so (3.1) and (3.2) imply

$$
\frac{\sin \xi}{1-a}-\frac{\sin \xi}{1-b}+\frac{\sin \xi}{1-c}-\frac{\sin \xi}{1-d}=-\left(\frac{\sin \xi}{1+a}-\frac{\sin \xi}{1+b}+\frac{\sin \xi}{1+c}-\frac{\sin \xi}{1+d}\right),
$$

and (3.3) gives

$$
\frac{l}{l^{2}+a^{2}}-\frac{l}{l^{2}+b^{2}}+\frac{l}{l^{2}+c^{2}}-\frac{l}{l^{2}+d^{2}}=0 .
$$

After some simplifications of these we arrive at

$$
1-a^{2}=\frac{\left(1-b^{2}\right)\left(1-c^{2}\right)\left(1-d^{2}\right)}{\left(1-c^{2}\right)\left(1-d^{2}\right)-\left(1-b^{2}\right)\left(1-d^{2}\right)+\left(1-b^{2}\right)\left(1-c^{2}\right)}
$$

and

$$
l^{2}+a^{2}=\frac{\left(l^{2}+b^{2}\right)\left(l^{2}+c^{2}\right)\left(l^{2}+d^{2}\right)}{\left(l^{2}+c^{2}\right)\left(l^{2}+d^{2}\right)-\left(l^{2}+b^{2}\right)\left(l^{2}+d^{2}\right)+\left(l^{2}+b^{2}\right)\left(l^{2}+c^{2}\right)} .
$$

The sum of these two equations is

$$
\begin{aligned}
1+l^{2}= & \left(1-a^{2}\right)+\left(l^{2}+a^{2}\right) \\
= & \frac{\left(1-b^{2}\right)\left(1-c^{2}\right)\left(1-d^{2}\right)}{\left(1-c^{2}\right)\left(1-d^{2}\right)-\left(1-b^{2}\right)\left(1-d^{2}\right)+\left(1-b^{2}\right)\left(1-c^{2}\right)}+ \\
& +\frac{\left(l^{2}+b^{2}\right)\left(l^{2}+c^{2}\right)\left(l^{2}+d^{2}\right)}{\left(l^{2}+c^{2}\right)\left(l^{2}+d^{2}\right)-\left(l^{2}+b^{2}\right)\left(l^{2}+d^{2}\right)+\left(l^{2}+b^{2}\right)\left(l^{2}+c^{2}\right)} .
\end{aligned}
$$

Multiplying by the denominators

$$
\begin{aligned}
&\left(1+l^{2}\right) {\left[\left(l^{2}+c^{2}\right)\left(l^{2}+d^{2}\right)+\left(l^{2}+b^{2}\right)\left(c^{2}-d^{2}\right)\right] \times } \\
& \times\left[\left(1-c^{2}\right)\left(1-d^{2}\right)+\left(1-b^{2}\right)\left(d^{2}-c^{2}\right)\right] \\
&=\left(1-b^{2}\right)\left(1-c^{2}\right)\left(1-d^{2}\right)\left[\left(l^{2}+c^{2}\right)\left(l^{2}+d^{2}\right)+\left(l^{2}+b^{2}\right)\left(c^{2}-d^{2}\right)\right]+ \\
&+\left(l^{2}+b^{2}\right)\left(l^{2}+c^{2}\right)\left(l^{2}+d^{2}\right)\left[\left(1-c^{2}\right)\left(1-d^{2}\right)+\left(1-b^{2}\right)\left(d^{2}-c^{2}\right)\right]
\end{aligned}
$$

is obtained. After multiplication on the left and rearranging on the right, we see

$$
\begin{aligned}
& \left(1+l^{2}\right)\left(1-c^{2}\right)\left(1-d^{2}\right)\left(l^{2}+c^{2}\right)\left(l^{2}+d^{2}\right)+ \\
& \quad+\left(1+l^{2}\right)\left(1-c^{2}\right)\left(1-d^{2}\right)\left(l^{2}+b^{2}\right)\left(c^{2}-d^{2}\right)+ \\
& \quad+\left(1+l^{2}\right)\left(1-b^{2}\right)\left(d^{2}-c^{2}\right)\left(l^{2}+c^{2}\right)\left(l^{2}+d^{2}\right)+ \\
& \quad+\left(1+l^{2}\right)\left(1-b^{2}\right)\left(d^{2}-c^{2}\right)\left(l^{2}+b^{2}\right)\left(c^{2}-d^{2}\right) \\
& =\left(1-b^{2}\right)\left(l^{2}+b^{2}\right)\left(c^{2}-d^{2}\right)\left[\left(1-c^{2}\right)\left(1-d^{2}\right)-\left(l^{2}+c^{2}\right)\left(l^{2}+d^{2}\right)\right]+ \\
& \quad+\left(1-c^{2}\right)\left(1-d^{2}\right)\left(l^{2}+c^{2}\right)\left(l^{2}+d^{2}\right)\left(1+l^{2}\right) .
\end{aligned}
$$


Rearranging to zero leads to

$$
\begin{aligned}
0= & -\left(1-b^{2}\right)\left(l^{2}+b^{2}\right)\left(c^{2}-d^{2}\right)\left[\left(1-c^{2}\right)\left(1-d^{2}\right)-\left(l^{2}+c^{2}\right)\left(l^{2}+d^{2}\right)\right]+ \\
& +\left(1+l^{2}\right)\left(1-c^{2}\right)\left(1-d^{2}\right)\left(l^{2}+b^{2}\right)\left(c^{2}-d^{2}\right)+ \\
& +\left(1+l^{2}\right)\left(1-b^{2}\right)\left(d^{2}-c^{2}\right)\left(l^{2}+c^{2}\right)\left(l^{2}+d^{2}\right)+ \\
& +\left(1+l^{2}\right)\left(1-b^{2}\right)\left(d^{2}-c^{2}\right)\left(l^{2}+b^{2}\right)\left(c^{2}-d^{2}\right)
\end{aligned}
$$

and finally we arrive at

$$
\begin{aligned}
0= & \left(1-b^{2}\right)\left(l^{2}+b^{2}\right)\left(c^{2}-d^{2}\right)\left(l^{2}+1\right)\left(2 d^{2}+l^{2}-1\right)+ \\
& +\left(1+l^{2}\right)\left(c^{2}-d^{2}\right)\left[\left(1-c^{2}\right)\left(1-d^{2}\right)\left(l^{2}+b^{2}\right)-\left(1-b^{2}\right)\left(l^{2}+c^{2}\right)\left(l^{2}+d^{2}\right)\right],
\end{aligned}
$$

that simplifies to

$$
0=\left(b^{2}-c^{2}\right)\left(l^{2}\left(b^{2}+d^{2}-2\right)-\left(b^{2}+d^{2}-2 b^{2} d^{2}\right)\right)\left(c^{2}-d^{2}\right)\left(1+l^{2}\right) .
$$

The second factor of this product is always negative because $b^{2}+d^{2}-2<0$ and $b^{2}+d^{2}>2 b^{2} d^{2}$, the fourth factor obviously positive, therefore $b= \pm c$ or $c= \pm d$ follows. If $b= \pm c$ then substituting it into (3.4) gives $a= \pm d$.

If $b=-c$ and $a=-d$, we get segments symmetrical to the midpoint of the chord.

If $b=c$ and $a=-d$, or $b=-c$ and $a=d$, then we have $-1<a<b=c<d=-a<1$ and $-1<c<d=a<$ $b=-c<1$, hence $\mathcal{S}_{1}$ and $\mathcal{S}_{2}$ are only subsegments of a segments that are not considered as two segments.

Since $a<b$ and $c<d$ the case $b=c$ and $a=d$ cannot happen.

If $c=-d$, then $a=-b$, which determines two segments equally symmetrical to the midpoint of the chord.

The proof of the theorem is complete.

\section{References}

[1] Kincses, J. and Kurusa, Á. : Can you recognise the shape of a figure by its shadows?, Beiträge zur Alg. und Geom., 36, 25-35 (1995).

[2] Kurusa, Á. : Visual distinguishability of segments, Int. Electron. J. Geom., 6, 56-67 (2013).

[3] Kurusa, Á. : Visual distinguishability of polygons, Beiträge zur Alg. und Geom., 54 , 659-667 (2013), https:/ / doi.org/10.1007/s13366-012-0121-7

[4] Kurusa, Á. : You can recognize the shape of a figure by its shadows!, Geom. Dedicata, 59, 113-125 (1996); https://doi.org/10.1007/BF00155723

[5] Kurusa, Á. : Can you see the bubbles in a foam?, Acta Sci. Math. (Szeged) 82, 663-694 (2016); https:/ / doi.org/10.14232/actasm-015-299-1

[6] Lukács, P. : Szakaszok takarási száma, Polygon, xxiv, 29-42 (in Hungarian) (2017).

\section{Affiliations}

PÉTER LUKÁCS

AdDRESS: University of Szeged, Dept. of Geometry, 6725, Szeged-Hungary.

E-MAIL: lukacsp@math.u-szeged.hu

ORCID ID:0000-0001-5790-97150 